\title{
A STRICT MAXIMUM THEOREM FOR ONE-PART FUNCTION SPACES AND ALGEBRAS
}

\author{
BY H. S. BEAR ${ }^{1}$ \\ Communicated by W. Rudin, March 17, 1964
}

The idea of a "part" of the spectrum of a function algebra was introduced by Gleason in [3]. Recently Bishop [2] extended one of the results of [3] and showed that any two points in one part of a function algebra have representing measures which are mutually absolutely continuous. Motivated by Bishop's work, we have shown [1] that the concept of a part extends to arbitrary linear spaces of functions in a way which generalizes the idea for an algebra. Our purpose here is to show how this concept can be used to prove a strict maximum theorem for subalgebras or subspaces.

Let $A$ be a separating, uniformly closed subalgebra of $C_{c}(X)$, all continuous complex-valued functions on a compact space $X$, and let $S_{A}$ denote the space of nonzero homomorphisms of $A$. Then $S_{A}$ is a compact subset of $A^{*}$ with the $w^{*}$ topology, and $X$ is homeomorphically embedded in $S_{A}$ as the set of evaluation functionals. The functions in $A$ are generally regarded as being extended from $X$ to continuous functions on all of $S_{A}$. If $A^{*}$ is given the norm topology rather than the customary $w^{*}$ topology, then the relation $x \sim y\left(x, y \in S_{A}\right)$ defined by $\|x-y\|<2$ is an equivalence relation on $S_{A}$, and the equivalence classes are called the Gleason parts of $S_{A}$.

Now let $C_{r}(X)$ be all continuous real functions on $X$, and let $B$ be a separating subspace of $C_{r}(X)$ which contains the constants. The parts of $X$ (with respect to $B$ ) are defined to be the equivalence classes under the relation $x \sim y$ defined by the condition that there exist a real number $a>1$ such that $1 / a<u(x) / u(y)<a$ for all positive functions $u \in B$. In [1] it is shown that if $B=\operatorname{Re} A$ for a function algebra $A$, then the $B$-parts coincide with the $A$-parts. We obtain a geometric interpretation of a part by regarding $X$ as embedded in $B^{*}$, and representing $B$ as the weak dual of $B^{*}$, restricted to the closed convex hull $T_{B}$ of $X$ in $B^{*}$. The parts of $T_{B}$, regarding $B$ as a subspace of $C_{r}\left(T_{B}\right)$, are (see [1]) those (necessarily convex) subsets $\Pi$ of $T_{B}$ which do not contain any extreme points of $T_{B}$, and have the property that if $x, y \in \Pi$, then the line in $B^{*}$ joining $x$ and $y$ intersects $T_{B}$ in a segment containing $x$ and $y$ in its interior. The parts of

1 This research was supported in part by Grant NSF-GP-216 of the National Science Foundation. 
$X$, considered as a subset of $T_{B}$, are the intersections of $X$ with the parts of $T_{B}$.

We can now state our maximum theorem.

THEOREM. If $B$ is a separating subspace of $C_{r}(X)$ containing the constants, $\Gamma$ is the Silov boundary of $B$ in $X$, and $X \sim \Gamma$ is a single part, then no function in $B$ assumes its maximum or minimum at a point of $X \sim \Gamma$ unless constant on $X \sim \Gamma$.

Proof. Let us consider $X$ as embedded in $B^{*}$ and let $T_{B}=\langle X\rangle$. Then $B$ is isometrically isomorphic to the continuous linear functionals on $B^{*}$, restricted to $T_{B}$, and $\Gamma$ is the closure of the extreme points of $T_{B}$. Our hypothesis is that $\Delta=X \sim \Gamma$ is contained in a single part $\Pi$ of $T_{B}$. Let $u$ be a nonconstant function in $B$, with $u(x) \neq u(y)$ and $x, y \in \Delta$. Then the line joining the functionals $x$ and $y$ of $B^{*}$ extends beyond $x$ and $y$ in $\Pi$. This segment need not (probably does not) intersect $X$ other than at $x$ and $y$. However, $u$ is linear on $T_{B}$, so $u(z)>u(x)$ for some $z \in \Pi$ on the line joining $x$ and $y$. The maximum of the linear functional $u$ on the convex set $T_{B}$ will be assumed at an extreme point $\theta \in \Gamma$, and hence $\|u\|=u(\theta) \geqq u(z)>u(x)$. Since $x$ is any point of $\Delta$, the theorem is proved.

If $A$ is a function algebra and some $f \in A$ assumes its maximum modulus at $x$, then some $u \in \operatorname{Re} A$ assumes its maximum at $x$. Hence we have the following corollary.

Corollary. If $A$ is a function algebra, $\Gamma$ is the Silov boundary of $A$ in its spectrum $S_{A}$, and $S_{A} \sim \Gamma$ is one part, then no function in $A$ assumes its maximum modulus at a point of $S_{A} \sim \Gamma$ unless constant on $S_{A} \sim \Gamma$.

\section{REFERENCES}

1. H. S. Bear, A geometric characterization of Gleason parts, Proc. Amer. Math. Soc. (to appear).

2. Errett Bishop, Representing measures for points in a uniform algebra, Bull. Amer. Math. Soc. 70 (1964), 121-122.

3. A. M. Gleason, Function algebras, Seminars and Analytic Functions, vol. 2, pp. 213-226, Inst. for Advanced Study, Princeton, N. J., 1957.

University of California, Santa Barbara 